Univerzitet u Beogradu
Poljoprivredni fakultet
Institut za poljoprivrednu tehniku
Naučni časopis
POLJOPRIVREDNA TEHNIKA
Godina XLV
Broj 1, 2020.
Strane: $51-64$

\title{
SIMPLE REGRESSION RELATIONSHIPS FOR ASSESSING THE PERFORMANCES OF SELECTED TILLAGE IMPLEMENTS IN SOUTH-EAST, NIGERIA
}

\author{
Okechukwu Oduma $^{* 1}$, Chineze G. Okeke ${ }^{2}$, Brendan E. Eje ${ }^{2}$, \\ Goziechukwu Inekwe ${ }^{1}$, Daniel C. Nnadi ${ }^{3}$ \\ ${ }^{1}$ Department of Agricultural \& Bioresources Engineering, \\ Michael Okpara University of Agriculture, Umudike, Nigeria \\ ${ }^{2}$ Department of Agricultural \& Bioresources Engineering \\ Enugu State University of Science and Technology \\ ${ }^{3}$ Department of Mechanical Engineering, \\ Michael Okpara University of Agriculture, Umudike, Nigeria
}

\begin{abstract}
Research was conducted on the three dominant agricultural soils of southeast Nigeria to develop some empirical regression relationships for predicting the performances of some selected tractor-hitched tillage implements from the experimental results obtained in the field. Results of the experiments revealed that the optimum speed of plough in clay-loam and loamy - sandy soil was $6 \mathrm{~km} / \mathrm{h}$ with corresponding field efficiencies of $88.11 \%$ and $87.55 \%$ respectively, while in sandy - clay soil, the plough recorded its optimum speed of $7 \mathrm{~km} / \mathrm{h}$ with field efficiency of $87.78 \%$. The optimum speed of harrow in clay-loam soil was $8 \mathrm{~km} / \mathrm{h}$ with field efficiency of $87.98 \%$. In loamy sandy soil, its optimum speed was $8 \mathrm{~km} / \mathrm{h}$ with field efficiency of $87.19 \%$; while in sandy - clay soil; it recorded optimum speed of $9 \mathrm{~km} / \mathrm{h}$ with field efficiency of $98.54 \%$. The optimum speed of ridger was $9 \mathrm{~km} / \mathrm{h}$ for all the soils with corresponding field efficiencies of $87.96 \%, 87.95 \%$, and $89.09 \%$ respectively, for clay-loam, loamy-sandy and sandy clay soil.
\end{abstract}

*Corresponding Author. E-mail.odumaoke@gmail.com 
The optimum speed of rotovator was also $9 \mathrm{~km} / \mathrm{hr}$ in all the soils with corresponding field efficiencies of $89.81 \%, 87.11 \%$, and $89.40 \%$ in clay-loam, loamy-sandy and sandy -clay soil, respectively.

These field efficiencies experimentally obtained in the field were compared with the efficiencies obtained from the regression equations using percentage error and the accuracy of the predictions were tested using error root mean square.

The comparison of the predicted results with the experimental results revealed that the regression equations broadly did not over or under- predict the experimental results, thus, the prediction errors were within allowable range of $\pm 5 \%$. The coefficient of determination, $\mathrm{R}^{2}$ for the regression equations developed for predicting the various performance indicators of the tractor - hitched implements vary from 0.7 to 0.9 which show that the variables tested were highly correlated and also an indication that the regression equations were adequate for predicting the performance of the implements.

Key words: Empirical, equation, performance, regression, tillage implement.

\section{INTRODUCTION}

Performance efficiencies/capabilities of farm machinery can generally be evaluated by the rate at which they carry out their field operations and the quality and quantity of production. [1] state that machine field capacity is the rate at which the machine can cover a given field operation within the limit of time. [2] noted that effective capacity is evaluated by the rate of working of implement measured in hectares per hour, and that the indicators involved are the implement operation width and the working speed with the allowance for time loss, in turning at the end of the field, adjustment and servicing of the machines. The efficiency of machines/implements indicates how good the machine can perform its functions. According to [3], an experienced farmer is usually conscious of the effective and efficient operation of his/her farm machinery because poor operation or improper utilization of the equipment may lead to great operating loss and minimizes production or result to total loss of money/productivity.

Differences exist among various agro ecological areas; and performance data from various tractor - hitched implements are essential document that guides farm machinery users and managers in proper selection of machinery for a given field operation under different soil types/conditions. Selection and utilization of farm machinery are better done with the aid of detailed performance information of such machinery taking into account the variations in agro ecological soil types/conditions. [4] noted that, due to some differences in the agro-ecological soil conditions, performance data of the field capacities of machines under varying soil type/conditions is very essential for machinery selection; the performance data are the essential parameters for assessing the performances of farm machinery. But regrettably this information are not provided to farmers or farm managers in Nigeria by the producers of the machines to guide the farmers in assessing and making proper selection of the machine/implement before buying them. [5] studied the performance efficiencies of chisel and disc plough and the impact they have on some physical properties of soil in Sudan. They came out with the performance data of these implements. However, their study was only on plough tested on only one soil type, other tillage implements and their power requirements were not 
considered in their study; thereafter, they recommended that more investigations are needed to verify the impact of the ploughs under study on the physical properties of different types of soils in the area.

[6] evaluated the performance of some selected tillage implements in Samaru, Zaria. The study only considered one soil type; and because of variations among soil types in an ecological area, results of such study cannot provide enough information that may guide farmers in selecting machines for their agricultural field operations.

[7] investigated the performance efficiency of tractor - hitched tillage tools in clay soil in Urmia, Iran. This study was also based on only one soil type and because of the same reason, the results may not guide farmers properly in machine selection to be used in other soil types; furthermore, Iran and South-east Nigeria may not have the same soil type with same properties/conditions; there must be differences; in which case, farmers in Nigeria may not use data from such study area to select their farm machinery.

[8] evaluated disc plough performance on sandy- loam soil at different moisture levels in Ilorin. In this study, he used dimensional analysis to develop model expressions relating the cutting depth, disc plough weight and draught force on sandy-loam soil. Despite the detailed study, did not consider the energy requirements of the implement for operation; and the study was conducted on only one soil type with only one tillage implement. These may not be enough to guide machine users in implement selection because of variations in soil type and conditions as emphasized earlier.

Development of empirical model is an essential and simple way of assisting the farmers, farm managers and other users of agricultural machinery both at subsistence and commercial level in assessing and predicting the possible performance capabilities of farm machinery in order to make proper selection of the equipment based on soil type/conditions and season of operation before purchasing and/or engaging any machine to work. This will go a long way to reduce failures, unnecessary break down, mismatching of implement to prime movers, minimize fuel consumption (energy loss), reduce cost and generally maximize production and profit [9]. The objective of this work is to develop empirical regression equations/relationships that will be used to predict the field performances of tractor hitched tillage implement in South-east agro- ecological region of Nigeria.

\section{MATERIALS AND METHOD}

\section{Description of the experimental site}

The experimental sites have average area of $8100 \mathrm{~m}^{2}(0.81 \mathrm{ha})$ each. The land area was divided into four units of $45 \times 45 \mathrm{~m}^{2}$ each for random observations. Each unit was separated by a distance of $2.5 \mathrm{~m}$ from the other to avoid interaction between the plot borders and to be equally used as head lands for the commencement of the experimental operations.

The tests were conducted in May, through June, July, August, September and October, 2016. These months coincide with planting season of the year; and will also offer the tractor and the hitched implements an exposure to wide range of soil conditions. 


\section{Description of Machine used and its operation}

A Massey Ferguson tractor with 3- point hitch systems and age of 5months from date of first hand purchase was hired with the hitched implements and used for the study. The same operator was used to operate the machine throughout the test to ensure minimal variation in the operation skill and style throughout the study.

Each field operation (test) starts in the morning $(9 \mathrm{am})$ and lasts for $2,5 \mathrm{~h}$ to ensure that the operator did not get weary during the operation and the machines are properly checked for faults before being engaged to work. This is to minimize delays or unnecessary failures and to ensure optimum production time during operation.

\section{Determination of soil physical properties}

Some soil physical and mechanical properties such as moisture contents, bulk density, soil structure, texture, porosity, cone index, penetration resistance and shear resistance which affect implement performances were examined before conducting the test, using the method adopted by [9].

\section{Field Performance Characteristics Test}

The field operations were generally performed longitudinally at selected forward speeds, the distance travelled and the corresponding time taken to complete the working distance were noted; and the total productive and delay time were evaluated and recorded [10]. The speed selections were made within the speed range recommended by [11] for tillage. The implement performance indicators such as field efficiency, effective field capacity, theoretical field capacity and material efficiency were evaluated.

\section{Measurement of Productive and Delay (Idle) Time}

The total time spent on the entire row length operation and the delay or idle time encountered in the operation which include, time for refilling the tank, time for repair of breakdown/adjustments, turning time, and any other idle time observed was noted and the actual time (productive time) used in the operation was evaluated from the relationship [3];

$$
\mathrm{T}_{\mathrm{e}}=\mathrm{T}_{\mathrm{t}}-\mathrm{T}_{\mathrm{d}}
$$

Where:

$\mathrm{T}_{\mathrm{e}}=$ actual (productive) time, $(\mathrm{h})$

$\mathrm{T}_{\mathrm{t}}=$ total time spent on the entire row length operation, (h)

$\mathrm{T}_{\mathrm{d}=}$ delay (idle) time, $(\mathrm{h})$ 
Measurement of turning time commences immediately the implement is raised on the completion of a row length, to initiate a turn until it turns completely to continue the operation.

\section{Determination Field Efficiency}

The field efficiency was determined from the expression suggested by [12]

$$
\varepsilon=\frac{100 T e}{T t}
$$

Where:

$\varepsilon=$ field efficiency, $\%$

$\mathrm{T}_{\mathrm{e}}=$ actual working (productive) time, $(\mathrm{h})$

$\mathrm{T}_{\mathrm{t}}=$ total working time $=\left(\mathrm{T}_{\mathrm{e}}+\mathrm{T}_{\mathrm{d}}\right),(\mathrm{h})$

$\mathrm{T}_{\mathrm{d}}=$ delay or idle time

\section{Determination of the Effective Field Capacity}

The effective field capacity was determined by noting the speed of operation, implements working width and the field efficiency of the machine; and then was evaluated from the expression suggested by [11]

$$
\mathrm{Ce}=\frac{s w e}{c}
$$

Where:

$\mathrm{Ce}=$ effective field capacity, ha/h $[\mathrm{a} / \mathrm{h}]$

$\mathrm{S}=$ speed $, \mathrm{km} / \mathrm{h}, \quad[\mathrm{mi} / \mathrm{h}]$

$\mathrm{W}=$ rated width of implement, $\mathrm{m}[\mathrm{ft}]$

$\mathrm{e}=$ field efficiency as a decimal

$\mathrm{c}=$ constant, $10[8.25]$

\section{Determination of Theoretical Field Capacity}

The Theoretical Field Capacity was determined by rearranging the expression suggested by [1] for field efficiency as follows

$$
\varepsilon=\frac{C e}{C t}
$$

By rearrangement,

$$
C_{t}=\frac{C e}{\varepsilon}
$$

Where:

$C_{t}=$ theoretical field capacity, ha/h

$\mathrm{Ce}=$ effective field capacity, ha/h

$\varepsilon=$ field efficiency, decimal 


\section{Determination of the Material Capacity}

The machine material capacity was determined by noting the speed of operation, implement working width, the field efficiency of the machine and the weight of soil scooped ( for tillage implements), but for the planter, the quantity/weight of seeds loaded in the hopper; and then was obtained from the expression.

$$
\mathrm{M}=\frac{\text { Swey }}{c}
$$

Where:

$\mathrm{M}=$ material capacity, $\mathrm{kg} / \mathrm{h}$

$\mathrm{y}=$ yield $/ \mathrm{mass}$ of material handled, $\mathrm{kg} / \mathrm{m}^{2}$

$\mathrm{s}=$ implement $/$ machine speed, $\mathrm{km} / \mathrm{h}$

$\mathrm{w}=$ implement working width, $\mathrm{cm}$

$\mathrm{e}=$ implement field efficiency, \%

$\mathrm{c}=$ constant $=10$

\section{Development of Empirical Regression Equation}

The data obtained from the study were subjected to regression analysis and empirical regression equations for predicting the implement performances at different operation speeds were developed.

\section{Determination of the adequacy of the equations}

The adequacy of the equations developed from the study were determined by comparing the results obtained from the experiment with the regression results using percentage error (eqn. 7) suggested by [13]

$$
\text { Error }=\frac{\text { Regression Result }- \text { Experimental Result }}{\text { Experimental Result }} \times 100
$$

Thereafter, the root mean square (RMS) of the error where evaluated to determine the accuracy of the predicted results. The predictions are considered accurate if the RMS errors of the prediction are within the tolerable limit of $\pm 5 \%$.

More so, the coefficient of determination, $\mathrm{R}^{2}$ will also indicate the adequacy of the model if it is within limit of 0 and 1 [14]. 


\section{RESULTS AND DISCUSSION}

Results of this research work are presented in Table 1-9

Table 1. Optimum speeds and efficiencies of tractor-hitched implements under different soil type/conditions

\begin{tabular}{cccccc}
\hline $\begin{array}{c}\text { Soil type/M.C, } \\
\%(w b)\end{array}$ & $\begin{array}{c}\text { Speed range, } \\
\mathrm{km} / \mathrm{h}\end{array}$ & \multicolumn{3}{c}{ Range and optimum values of field efficiency, (\%) } \\
\cline { 3 - 6 } & & Plough & Harrow & Ridger & Rotovator \\
Clay-loam & $5-10$ & $85.74-88.11$ & $82.59-87.98$ & $83.65-87.96$ & $85.81-89.81$ \\
& Optimum speed & $6 \mathrm{~km} / \mathrm{h}$ & $8 \mathrm{~km} / \mathrm{h}$ & $9 \mathrm{~km} / \mathrm{h}$ & $9 \mathrm{~km} / \mathrm{h}$ \\
M.c,\% $(w b)$ & & $(15.5-17.2)$ & $(14.8-16.2)$ & $(14.0-14.4)$ & $(13.0-14.2)$ \\
Loamy-sandy & $5-10$ & $85.31-87.55$ & $83.41-87.19$ & $85.54-87.95$ & $81.10-87.11$ \\
M.c, \% $(w b)$ & Optimum speed & $6 \mathrm{~km} / \mathrm{h}$ & $8 \mathrm{~km} / \mathrm{h}$ & $9 \mathrm{~km} / \mathrm{h}$ & $9 \mathrm{~km} / \mathrm{h}$ \\
& & $(15.2-16.2)$ & $(13.3-15.4)$ & $(13.2-14.5)$ & $(13.1-13.6)$ \\
Sandy-clay & $5-10$ & $85.90-87.98$ & $87.05-98.54$ & $86.26-89.09$ & $87.05-89.40$ \\
M.c, \% $(w b)$ & Optimum speed & $7 \mathrm{~km} / \mathrm{h}$ & $9 \mathrm{~km} / \mathrm{h}$ & $9 \mathrm{~km} / \mathrm{h}$ & $9 \mathrm{~km} / \mathrm{h}$ \\
& & $(14.8-18.6)$ & $(13.0-19.3)$ & $(13.0-17.1)$ & $(13.3-16.3)$ \\
\hline
\end{tabular}

Table 2. Regression equations for predicting ploughing efficiencies of the implement in different soil types in south-east Nigeria

\begin{tabular}{cccc}
\hline Soil type & Efficiences (performance indicators) & Regression equations & $\begin{array}{c}\text { Coefficient of } \\
\text { determination, } R^{2}\end{array}$ \\
\hline Clay- & Field efficiency $(\mathcal{E})$ & $Y=0.067 x^{2}-0.7733 x+89.283$ & 0.9838 \\
loam & Effective capacity $(\mathrm{Ce})$ & $Y=0.0864 x^{2}-1.4406 x+6.8173$ & 0.8081 \\
& Theoretical field capacity $($ Te $)$ & $Y=0.0026 x^{2}-0.0877 x+1.7641$ & 0.909 \\
Loamy- & Material efficiency $($ Me $)$ & $Y=0.0888 x^{2}-1.6513 x+49.11$ & 0.71 \\
sand & Field efficiency $(\mathcal{E})$ & $Y=-0.089 x^{2}+0.8208 x+85.801$ & 0.9615 \\
& Effective capacity $(\mathrm{Ce})$ & $Y=-0.0036 x^{2}+0.0318 x+0.9189$ & 0.9524 \\
Sandy - & Theoretical field capacity $($ Te $)$ & $Y=0.0019 x^{2}-0.033 x+1.2483$ & 0.8473 \\
clay & Material efficiency $(\mathrm{Me})$ & $Y=-0.2184 x^{2}+2.911 x+32.207$ & 0.9501 \\
& Field efficiency $(\mathcal{E})$ & $Y=0.0198 x^{2}+0.0847 x+84.957$ & 0.9258 \\
& Effective capacity $(\mathrm{Ce})$ & $Y=-0.0209 x^{2}-1.1748 x+6.0411$ & 0.8427 \\
& Theoretical field capacity $($ Te $)$ & $Y=-0.9018 x^{2}+13.699 x+0.1481$ & 0.9625 \\
\hline Material efficiency $(\mathrm{Me})$ & & 0.9515
\end{tabular}

Note: $Y=$ Performance indicators; $X=$ Operational speeds

Table 3. Regression equations for predicting the harrowing efficiencies of the implementin different types in south-east zone.

\begin{tabular}{|c|c|c|c|}
\hline Soil type & Efficiencies (performance indicators) & Regression Equations & $\begin{array}{c}\text { Coefficient of } \\
\text { determination, } R^{2}\end{array}$ \\
\hline $\begin{array}{l}\text { Clay- } \\
\text { loam }\end{array}$ & $\begin{array}{l}\text { Field efficiency }(\mathcal{E}) \\
\text { Effective capacity }(\mathrm{Ce}) \\
\text { Theoretical field capacity }(\mathrm{Te}) \\
\text { Material efficiency }(\mathrm{Me})\end{array}$ & $\begin{array}{l}Y=-0.3745 x^{2}+7.4659 x+50.757 \\
Y=-0.0056 x^{2}+0.1486 x+0.3631 \\
Y=0.0041 x^{2}+0.0885 x+0.9156 \\
Y=0.1952 x^{2}-0.6095 x+84.343\end{array}$ & $\begin{array}{l}0.9149 \\
0.9007 \\
0.8894 \\
0.9144\end{array}$ \\
\hline $\begin{array}{l}\text { Sandy - } \\
\text { clay }\end{array}$ & $\begin{array}{l}\text { Field efficiency }(\mathcal{E}) \\
\text { Effective capacity }(\mathrm{Ce}) \\
\text { Theoretical field capacity }(\mathrm{Te}) \\
\text { Material efficiency }(\mathrm{Me}) \\
\text { Field efficiency }(\mathcal{E}) \\
\text { Effective capacity }(\mathrm{Ce}) \\
\text { Theoretical field capacity(Te) } \\
\text { Material efficiency }(\mathrm{Me})\end{array}$ & $\begin{array}{l}Y=-0.3423 x^{2}+5.2326 x+67.303 \\
Y=-0.0301 x^{2}+0.550 x-1.0181 \\
Y=0.0085 x^{2}+0.186 x+0.6977 \\
Y=-1.512 x^{2}+30.058 x-26.412 \\
Y=0.3945 x^{2}-4.2087 x+97.777 \\
Y=-0.0044 x^{2}+0.1375 x+0.4436 \\
Y=-0.01 x^{2}+0.194 x+0.5459 \\
Y=-0.3586 x^{2}+11.229 x+36.297\end{array}$ & $\begin{array}{l}0.9549 \\
0.9752 \\
0.9475 \\
0.9443 \\
0.8652 \\
0.9105 \\
0.9607 \\
0.9105\end{array}$ \\
\hline
\end{tabular}

Note: $Y=$ Performance indicators; $X=$ Operational speeds 
Table 4. Regression equations for predicting the ridging efficiencies of the implement in different soil types in south-east zone.

\begin{tabular}{|c|c|c|c|}
\hline Soil type & Efficiencies (performance indicators) & Regression equations & $\begin{array}{c}\text { Coefficient of } \\
\text { determination, } R^{2}\end{array}$ \\
\hline $\begin{array}{l}\text { Clay- } \\
\text { loam }\end{array}$ & $\begin{array}{l}\text { Field efficiency }(\mathcal{E}) \\
\text { Effective capacity }(\mathrm{Ce}) \\
\text { Theoretical field capacity }(\mathrm{Te}) \\
\text { Material efficiency }(\mathrm{Me})\end{array}$ & $\begin{array}{l}Y=-0.1873 x^{2}+3.9156 x+67.166 \\
Y=-0.0329 x^{2}+0.9125 x-4.1054 \\
Y=0.027 x^{2}-0.3873 x+2.5757 \\
Y=1.3063 x^{2}-17.514 x+115.46\end{array}$ & $\begin{array}{l}0.933 \\
0.9112 \\
0.8603 \\
0.9276\end{array}$ \\
\hline $\begin{array}{l}\text { Loamy- } \\
\text { sand }\end{array}$ & $\begin{array}{l}\text { Field efficiency (E) } \\
\text { Effective capacity }(\mathrm{Ce}) \\
\text { Theoretical field capacity }(\mathrm{Te}) \\
\text { Material efficiency }(\mathrm{Me}) \\
\text { Field efficiency }(\mathcal{E}) \\
\text { Effective capacity }(\mathrm{Ce}) \\
\text { Theoretical field capacity }(\mathrm{Te}) \\
\text { Material efficiency }(\mathrm{Me})\end{array}$ & $\begin{array}{l}Y=-0.1861 x^{2}+3.5461+70.901 \\
Y=-0.0061 x^{2}+0.114 x+0.6533 \\
Y=-0.0217 x^{2} 0.3893 x-0.3754 \\
Y=-1.1914 x^{2}+21.493 x-28.259 \\
Y=0.2082 x^{2}-3.1954 x+98.731 \\
Y=-0.0126 x^{2}+0.1867 x+0.6991 \\
Y=0.0019 x^{2}+0.0375 x+1.191 \\
Y=0.8154 x^{2}-11.968 x+109.01\end{array}$ & $\begin{array}{l}0.9637 \\
0.859 \\
0.8117 \\
0.899 \\
0.9425 \\
0.9591 \\
0.9118 \\
0.9233 \\
\end{array}$ \\
\hline
\end{tabular}

Note: $Y=$ Performance indicators; $X=$ Operational speeds

Table 5. Regression equations for predicting the pulverizing efficiencies of the implement in different soil types in south-east zone.

\begin{tabular}{lllc}
\hline $\begin{array}{l}\text { Soil } \\
\text { type }\end{array}$ & Efficiencies (performance indicators) & \multicolumn{1}{c}{ Regression equations } & $\begin{array}{c}\text { Coefficient of } \\
\text { determination, } R^{2}\end{array}$ \\
\hline Clay- & Field efficiency $(\mathcal{E})$ & $Y=-0.1741 x^{2}+4.531 x+59.272$ & 0.9672 \\
loam & Effective capacity $(\mathrm{Ce})$ & $Y=0.0033 x^{2}-0.0715 x+1.1462$ & 0.9501 \\
& Theoretical field capacity $(\mathrm{Te})$ & $Y=-0.0769 x^{2}+2.0311 x-11.388$ & 0.8773 \\
& Material efficiency $(\mathrm{Me})$ & $Y=-0.2234 x^{2}+5.8529 x-13.643$ & 0.9775 \\
Loamy- & & & 0.9122 \\
sand & Field efficiency (E) & $Y=-0.7563 x^{2}+18.097 x-19.798$ & 0.9614 \\
& Effective capacity $(\mathrm{Ce})$ & $Y=-0.0006 x^{2}+0.0113 x+0.7612$ & 0.9396 \\
& Theoretical field capacity $(T e)$ & $Y=-0.0812 x^{2}+1.668 x-6.5609$ & 0.8995 \\
Sandy- & Material efficiency $(\mathrm{Me})$ & $Y=-0.868 x^{2}+20.363 x-90.59$ & 0.9235 \\
clay & Field efficiency $(\mathcal{E})$ & $Y=-0.0825 x^{2}+1.9006 x+76.649$ & 0.9162 \\
& Effective capacity $(\mathrm{Ce})$ & $Y=-0.0053 x^{2}+0.1441 x-0.1535$ & 0.7867 \\
& Theoretical field capacity $(\mathrm{Te})$ & $Y=-0.0426 x^{2}+0.7346 x-1.1896$ & 0.8545 \\
\hline
\end{tabular}

Note: $Y=$ Performance indicators; $X=$ Operational speeds

Table 6. Comparison of the field experiment and regression equation results for ploughing operation

\begin{tabular}{|c|c|c|c|c|c|c|c|c|c|c|c|c|c|}
\hline \multirow[t]{2}{*}{ Soil type } & \multirow[t]{2}{*}{$\begin{array}{l}\text { speed } \\
\mathrm{km} / \mathrm{h}\end{array}$} & \multicolumn{3}{|c|}{ Field efficiency,\% } & \multicolumn{3}{|c|}{$\begin{array}{l}\text { Effective field } \\
\text { capacity, ha/h }\end{array}$} & \multicolumn{3}{|c|}{$\begin{array}{l}\text { Theoretical field } \\
\text { capacity, ha/h }\end{array}$} & \multicolumn{3}{|c|}{$\begin{array}{l}\text { Material efficiency, } \\
\mathrm{kg} / \mathrm{h}\end{array}$} \\
\hline & & Exp. & Regr. & $E r \%$ & Exp. & Regr & $E r \%$ & Exp. & Regr & $E r \%$ & $E x p$ & Regr & $E r \%$ \\
\hline \multirow{6}{*}{$\begin{array}{l}\text { Clay- } \\
\text { loam }\end{array}$} & 5 & 87.08 & 87.47 & 4.48 & 1.031 & 1.016 & -1.45 & 1.844 & 1.162 & -1.86 & 43.46 & 42.82 & -1.46 \\
\hline & 6 & 88.11 & 87.26 & -0.96 & 0.99 & 1.002 & 1.21 & 1.123 & 1.147 & 2.14 & 41.73 & 42.18 & 1.08 \\
\hline & 7 & 87.05 & 87.08 & 0.03 & 1.978 & 0.988 & -0.01 & 1.124 & 1.132 & 0.71 & 41.22 & 40.53 & -1.67 \\
\hline & 8 & 86.45 & 86.84 & -4.51 & 1.019 & 0.974 & -4.42 & 1.179 & 1.117 & -5.3 & 42.95 & 40.89 & -4.8 \\
\hline & 9 & 85.74 & 77.18 & -9.77 & 0.847 & 0.960 & 13.5 & 0.981 & 0.912 & -7.6 & 35.67 & 40.24 & 12.8 \\
\hline & $\begin{array}{l}10 \\
R M S\end{array}$ & 87.24 & 86.42 & $\begin{array}{l}-0.94 \\
4.48\end{array}$ & 1.002 & 0.946 & $\begin{array}{l}-5.6 \\
6.32\end{array}$ & 1.149 & 1.059 & $\begin{array}{l}-7.83 \\
5.01\end{array}$ & 42.23 & 38.35 & $\begin{array}{l}-9.19 \\
6.81\end{array}$ \\
\hline \multirow{6}{*}{$\begin{array}{l}\text { Loamy- } \\
\text { sandy }\end{array}$} & 5 & 86.79 & 88.99 & 2.52 & 0.981 & 0.976 & -0.51 & 1.130 & 1.120 & -0.88 & 41.35 & 40.76 & -1.42 \\
\hline & 6 & 88.55 & 88.79 & 1.40 & 0.991 & 0.958 & -3.33 & 1.119 & 1.104 & -1.34 & 41.77 & 40.50 & -3.04 \\
\hline & 7 & 87.07 & 88.59 & 1.75 & 0.875 & 0.955 & 9.14 & 1.005 & 1.088 & 8.26 & 36.88 & 40.24 & 9.11 \\
\hline & 8 & 85.13 & 88.40 & 3.84 & 0.932 & 0.922 & -1.07 & 1.102 & 1.072 & -2.72 & 40.30 & 39.26 & -2.58 \\
\hline & 9 & 86.02 & 88.21 & 2.55 & 0.911 & 0.904 & -0.77 & 1.111 & 1.056 & -4.95 & 41.09 & 39.73 & -3.31 \\
\hline & $\begin{array}{l}10 \\
R M S\end{array}$ & 82.31 & 88.02 & $\begin{array}{l}6.94 \\
3.71\end{array}$ & 0.885 & 0.886 & $\begin{array}{l}0.11 \\
4.00\end{array}$ & 1.003 & 1.040 & $\begin{array}{l}3.69 \\
4.42\end{array}$ & 39.32 & 39.47 & $\begin{array}{l}-0.38 \\
4.31\end{array}$ \\
\hline \multirow{6}{*}{$\begin{array}{l}\text { Sandy- } \\
\text { clay }\end{array}$} & 5 & 85.90 & 84.71 & -1.39 & 0.974 & 1.061 & 8.93 & 1.134 & 1.199 & 5.73 & 41.05 & 45.20 & 9.21 \\
\hline & 6 & 83.23 & 85.72 & 2.69 & 1.062 & 1.045 & -1.60 & 1.275 & 1.171 & -8.16 & 44.76 & 44.53 & -0.51 \\
\hline & 7 & 86.23 & 86.22 & -0.13 & 1.113 & 1.029 & -7.55 & 1.282 & 1.143 & -10.8 & 46.91 & 43.86 & -6.50 \\
\hline & 8 & 88.23 & 86.97 & $-1,43$ & 1.143 & 1.013 & -11.4 & 1.296 & 1.115 & -14.0 & 48.18 & 43.19 & -10.4 \\
\hline & 9 & 88.22 & 87.73 & -0.56 & 0.848 & 0.997 & 17.6 & 0.961 & 1.087 & 13.1 & 35.74 & 42.52 & 19.0 \\
\hline & 10 & 87.78 & 88.48 & 0.80 & 0.985 & 0.981 & -0.41 & 1.122 & 1.059 & -5.61 & 41.52 & 41.85 & 0.79 \\
\hline
\end{tabular}


Table 7. Comparison of the field experiment and regression equation results for harrowing operation

\begin{tabular}{|c|c|c|c|c|c|c|c|c|c|c|c|c|c|}
\hline \multirow[t]{2}{*}{$\begin{array}{l}\text { Soil } \\
\text { type }\end{array}$} & \multirow[t]{2}{*}{$\begin{array}{l}\text { Speed } \\
\mathrm{km} / \mathrm{h}\end{array}$} & \multicolumn{3}{|c|}{ Field efficiency,\% } & \multicolumn{3}{|c|}{$\begin{array}{l}\text { Effective field } \\
\text { capacity, ha/h }\end{array}$} & \multicolumn{3}{|c|}{$\begin{array}{c}\text { Theoretical field } \\
\text { capacity, ha/h }\end{array}$} & \multicolumn{3}{|c|}{$\begin{array}{c}\text { Material efficiency, } \\
\mathrm{kg} / \mathrm{h}\end{array}$} \\
\hline & & Exp. & Regr & Er\% & Exp. & Regr & $E r \%$ & Exp. & Regr & $E r \%$ & Exp & Regr & $E r \%$ \\
\hline \multirow{7}{*}{$\begin{array}{l}\text { Clay- } \\
\text { loam }\end{array}$} & 5 & 82.59 & 83.68 & 1.32 & 1.063 & 1.076 & 1.22 & 1.287 & 1.329 & 3.26 & 86.8992 .04 & 88.01 & 1.29 \\
\hline & 6 & 83.42 & 85.04 & 1.94 & 1.126 & 1.113 & -1.15 & 1.350 & 1.347 & -0.22 & 89.67 & 91.01 & -1.12 \\
\hline & 7 & 80.17 & 86.40 & 7.77 & 1.097 & 1.150 & 4.83 & 1.368 & 1.365 & -0.22 & 105.8 & 94.00 & 4.83 \\
\hline & 8 & 87.98 & 87.76 & -0.25 & 1.289 & 1.187 & -7.91 & 1.465 & 1.387 & -5.60 & 97.76 & 97.00 & -8.28 \\
\hline & 9 & 86.70 & 89.12 & 2.79 & 1.196 & 1.224 & 2.34 & 1.379 & 1.401 & 1.60 & 101.3 & 99.99 & 2.28 \\
\hline & 10 & 88.56 & 90.48 & 2.17 & 1.239 & 1.261 & 2.73 & 1.399 & 1.419 & 1.43 & & 102.9 & 1.68 \\
\hline & $R M S$ & & & 3.60 & & & 4.10 & & & 2.81 & & & 4.11 \\
\hline \multirow{7}{*}{$\begin{array}{l}\text { Loamy- } \\
\text { sandy }\end{array}$} & 5 & 83.58 & 85.68 & 2.51 & 1.192 & 1.302 & 9.23 & 1.426 & 1.517 & 6.38 & 97.43 & 108.7 & 11.5 \\
\hline & 6 & 86.82 & 85.41 & -1.62 & 1.389 & 1.327 & -4.46 & 1.600 & 1.542 & -3.63 & 113.5 & 106.6 & -6.14 \\
\hline & 7 & 87.08 & 85.17 & -2.19 & 1.436 & 1.352 & -5.85 & 1.649 & 1.567 & -4.97 & 117.4 & 104.5 & -11.0 \\
\hline & 8 & 84.19 & 84.92 & 0.87 & 1.399 & 1.377 & -1.57 & 1.589 & 1.592 & -0.19 & 98.32 & 102.4 & 4.13 \\
\hline & 9 & 55.22 & 84.24 & -1.15 & 1.386 & 1.402 & 1.15 & 1.603 & 1.617 & 0.89 & 97.18 & 100.3 & 3.20 \\
\hline & 10 & 83.41 & 84.44 & 1.23 & 1.394 & 1.427 & 2.37 & 1.611 & 1.642 & 1.92 & 96.74 & 98.20 & 1.51 \\
\hline & $R M S$ & & & 4.23 & & & 5.03 & & & 3.72 & & & 6.19 \\
\hline \multirow{6}{*}{$\begin{array}{l}\text { Sandy- } \\
\text { clay }\end{array}$} & 5 & 87.55 & 87.15 & -0.46 & 1.095 & 1.116 & -1.92 & 1.251 & 1.291 & 3.20 & 89.51 & 92.09 & 2.88 \\
\hline & 6 & 86.05 & 87.48 & 1.66 & 1.204 & 1.182 & -1.83 & 1.400 & 1.357 & -3.07 & 98.41 & 97.17 & -1.26 \\
\hline & 7 & 86.95 & 87.82 & -0.99 & 1.281 & 1.248 & -2.58 & 1.473 & 1.423 & -3.39 & 104.7 & 102.3 & -2.35 \\
\hline & 8 & 91.38 & 88.15 & -3.53 & 1.343 & 1.314 & -2.16 & 1.470 & 1.489 & 1.29 & 109.8 & 107.3 & -2.23 \\
\hline & 9 & 88.54 & 88.49 & -0.11 & 1.311 & 1.380 & 5.26 & 1.480 & 1.555 & 5.07 & 107.2 & 112.4 & 4.90 \\
\hline & $\begin{array}{c}10 \\
R M S\end{array}$ & 87.45 & 88.82 & $\begin{array}{l}1.57 \\
1.82\end{array}$ & 1.458 & 1.446 & $\begin{array}{l}-0.82 \\
2.83\end{array}$ & 1.667 & 1.621 & $\begin{array}{r}-2.76 \\
3.35\end{array}$ & 119.2 & 117.5 & $\begin{array}{r}-1.41 \\
2.84\end{array}$ \\
\hline
\end{tabular}

Table 8. Comparison of the field experiment and regression equation results for ridging operation

\begin{tabular}{|c|c|c|c|c|c|c|c|c|c|c|c|c|c|}
\hline \multirow[t]{2}{*}{$\begin{array}{l}\text { Soil } \\
\text { type }\end{array}$} & \multirow[t]{2}{*}{$\begin{array}{l}\begin{array}{l}\text { Speed, } \\
\mathrm{km} / \mathrm{h}\end{array} \\
\end{array}$} & \multicolumn{3}{|c|}{ Field efficiency, \% } & \multicolumn{3}{|c|}{$\begin{array}{l}\text { Effective field } \\
\text { capacity, ha/h }\end{array}$} & \multicolumn{2}{|c|}{$\begin{array}{l}\text { Theoretical field } \\
\text { capacity, ha/h }\end{array}$} & \multicolumn{4}{|c|}{$\begin{array}{c}\text { Material efficiency, } \\
\mathrm{kg} / \mathrm{h}\end{array}$} \\
\hline & & Exp. & Regr & $E r \%$ & Exp. & Regr & $E r \%$ & Exp. & Regr & $E r \%$ & $\operatorname{Exp}$ & Regr & $E r \%$ \\
\hline \multirow{7}{*}{$\begin{array}{l}\text { Clay- } \\
\text { loam }\end{array}$} & 5 & 83.65 & 83.68 & 0.04 & 1.065 & 1.117 & 4.88 & 1.273 & 1.152 & -9.51 & 59.85 & 62.57 & 4.55 \\
\hline & 6 & 85.68 & 84.56 & -1.31 & 0.932 & 1.113 & 19.4 & 1.088 & 1.200 & 10.3 & 52.38 & 62.12 & 18.6 \\
\hline & 7 & 83.90 & 85.43 & 1.83 & 1.457 & 1.109 & -23.9 & 1.260 & 1.248 & -0.95 & 79.40 & 61.67 & -22.3 \\
\hline & 8 & 86.00 & 86.31 & 0.36 & 1.152 & 1.105 & -4.08 & 1.340 & 1.296 & -3.28 & 64.74 & 61.22 & -5.44 \\
\hline & 9 & 88.06 & 87.18 & -0.10 & 0.945 & 1.101 & 16.5 & 1.073 & 1.344 & 25.3 & 53.11 & 60.77 & 14.4 \\
\hline & 10 & 87.92 & 88.05 & 0.15 & 1.089 & 1.100 & 0.73 & 1.603 & 1.392 & -13.2 & 59.19 & 60.32 & 1.91 \\
\hline & $R M S$ & & & 0.91 & & & 5.81 & & & 4.63 & & & 4.33 \\
\hline \multirow{7}{*}{$\begin{array}{l}\text { Loamy- } \\
\text { sandy }\end{array}$} & 5 & 87.54 & 87.59 & 5.37 & 1.007 & 1.087 & 7.94 & 1.150 & 1.249 & 8.61 & 56.59 & 61.07 & 7.92 \\
\hline & 6 & 87.45 & 87.50 & 0.06 & 1.175 & 1.109 & -5.62 & 1.344 & 1.265 & -5.88 & 66.04 & 61.20 & -6.12 \\
\hline & 7 & 87.54 & 87.42 & -1.37 & 1.166 & 1.131 & -3.00 & 1.332 & 1.281 & -3.83 & 65.55 & 62.92 & -4.01 \\
\hline & 8 & 87.48 & 87.39 & -0.10 & 1.170 & 1.153 & -1.45 & 1.290 & 1.297 & 0.54 & 63.18 & 63.85 & 1.05 \\
\hline & 9 & 86.95 & 87.26 & 0.36 & 1.165 & 1.175 & 0.86 & 1.318 & 1.313 & -0.38 & 64.39 & 64.77 & 0.59 \\
\hline & 10 & 87.32 & 87.17 & -12.8 & 1.169 & 1.195 & 3.27 & 1.301 & 1.329 & 2.15 & 64.52 & 65.69 & 1.82 \\
\hline & $R M S$ & & & 2.61 & & & 3.42 & & & 2.71 & & & 2.52 \\
\hline \multirow{7}{*}{$\begin{array}{l}\text { Sandy- } \\
\text { clay }\end{array}$} & 5 & 87.07 & 86.63 & -0.51 & 1.170 & 1.175 & 0.44 & 1.344 & 1.359 & 1.12 & 65.75 & 64.43 & -2.00 \\
\hline & 6 & 86.60 & 86.91 & 0.36 & 1.182 & 1.168 & -1.18 & 1.365 & 1.345 & -1.47 & 66.43 & 65.25 & -1.78 \\
\hline & 7 & 86.26 & 87.20 & 1.08 & 1.165 & 1.162 & -0.26 & 1.351 & 1.331 & -1.48 & 65.47 & 66.06 & 0.90 \\
\hline & 8 & 89.19 & 87.48 & -1.92 & 1.206 & 1.155 & -4.23 & 1.352 & 1.317 & -2.59 & 67.78 & 66.87 & -1.34 \\
\hline & 9 & 86.09 & 87.77 & 1.95 & 1.009 & 1.148 & 13.8 & 1.164 & 1.303 & 11.9 & 56.31 & 67.69 & 20.2 \\
\hline & 10 & 88.82 & 88.05 & -0.87 & 1.218 & 1.141 & -6.32 & 1.371 & 1.289 & 5.98 & 77.05 & 68.50 & -11.1 \\
\hline & $R M S$ & & & 0.64 & & & 3.12 & & & 4.12 & & & 4.22 \\
\hline
\end{tabular}


Table 9. Comparison of the field experiment and regression equation

\begin{tabular}{|c|c|c|c|c|c|c|c|c|c|c|c|c|c|}
\hline \multirow{2}{*}{$\begin{array}{l}\text { Soil } \\
\text { type }\end{array}$} & \multirow{2}{*}{$\begin{array}{l}\begin{array}{l}\text { Speed } \\
\mathrm{km} / \mathrm{h}\end{array} \\
\\
5\end{array}$} & \multicolumn{3}{|c|}{ Field efficiency, \% } & \multicolumn{3}{|c|}{$\begin{array}{l}\text { Effective field } \\
\text { capacity, ha/h }\end{array}$} & \multicolumn{3}{|c|}{$\begin{array}{c}\text { Theoretical field } \\
\text { capacity, ha/h }\end{array}$} & \multicolumn{3}{|c|}{$\begin{array}{l}\text { Material efficiency, } \\
\mathrm{kg} / \mathrm{hr}\end{array}$} \\
\hline & & Exp. & Regr & $E r \%$ & Exp. & $\operatorname{Regr}$ & $E r \%$ & Exp. & Regr & $E r \%$ & $\operatorname{Exp}$ & Regr & $E r \%$ \\
\hline \multirow{6}{*}{$\begin{array}{l}\text { Clay- } \\
\text { loam }\end{array}$} & 6 & 85.81 & 85.81 & 0.00 & 0.775 & 0.789 & 2.97 & 0.903 & 0.916 & 1.39 & 20.93 & 18.20 & -13.0 \\
\hline & 7 & 87.38 & 85.55 & -0.95 & 0.759 & 0.797 & 5.01 & 0.869 & 0.911 & 4.83 & 20.49 & 19.17 & -6.42 \\
\hline & 8 & 88.20 & 87.30 & -1.02 & 0.962 & 0.795 & -17.4 & 1.091 & 0.906 & -17.0 & 25.97 & 20.15 & -22.4 \\
\hline & 9 & 88.24 & 88.05 & -0.22 & 0.669 & 0.794 & 18.7 & 0.758 & 0.902 & 19.0 & 18.06 & 21.12 & 16.9 \\
\hline & 10 & 89.81 & 88.89 & -1.13 & 0.811 & 0.793 & -2.22 & 0.903 & 0.898 & -0.55 & 21.90 & 22.09 & 0.89 \\
\hline & $R M S$ & 86.63 & 89.54 & $\begin{array}{l}3.36 \\
1.22\end{array}$ & 0.795 & 0.792 & $\begin{array}{l}-0.38 \\
3.16\end{array}$ & 0.918 & 0.893 & $\begin{array}{l}-2.72 \\
5.02\end{array}$ & 21.47 & 23.06 & $\begin{array}{l}7.41 \\
4.18\end{array}$ \\
\hline \multirow{7}{*}{$\begin{array}{l}\text { Loamy } \\
\text {-sandy }\end{array}$} & 5 & 81.10 & 83.80 & 3.33 & 0.804 & 0.804 & 0.00 & 0.913 & 0.917 & 0.48 & 21.71 & 22.51 & 3.70 \\
\hline & 6 & 86.76 & 84.45 & -2.67 & 0.803 & 0.805 & 0.21 & 0.925 & 0.924 & -0.14 & 21.68 & 23.19 & 6.95 \\
\hline & 7 & 86.83 & 85.09 & -2.00 & 0.812 & 0.805 & -0.86 & 0.935 & 0.930 & -0.53 & 27.94 & 23.86 & -14.6 \\
\hline & 8 & 84.92 & 85.74 & 0.97 & 0.800 & 0.805 & 0.63 & 0.911 & 0.936 & 2.78 & 22.61 & 24.53 & 8.89 \\
\hline & 9 & 87.11 & 86.39 & -0.83 & 0.811 & 0.806 & -0.65 & 1.002 & 0.943 & -5.92 & 26.81 & 25.21 & -6.36 \\
\hline & 10 & 85.81 & 87.04 & 1.43 & 0.804 & 0.806 & 0.26 & 0.916 & 0.949 & 3.61 & 24.41 & 25.89 & 6.01 \\
\hline & $R M S$ & & & 2.24 & & & 0.51 & & & 2.21 & & & 3.60 \\
\hline \multirow{7}{*}{$\begin{array}{l}\text { Sandy- } \\
\text { clay }\end{array}$} & 5 & 87.05 & 87.81 & 0.87 & 0.800 & 0.763 & -4.61 & 0.919 & 0.900 & -2.05 & 21.60 & 20.75 & -3.95 \\
\hline & 6 & 89.40 & 87.91 & -1.67 & 0.778 & 0.776 & -0.23 & 0.933 & 0.910 & -2.42 & 21.01 & 21.10 & 0.45 \\
\hline & 7 & 86.88 & 88.02 & 1.31 & 0.685 & 0.789 & 15.2 & 0.788 & 0.921 & 15.7 & 18.50 & 21.46 & 16.0 \\
\hline & 8 & 87.51 & 88.12 & 0.70 & 0.902 & 1.274 & 41.2 & 1.031 & 0.931 & -9.72 & 24.35 & 21.82 & -10.4 \\
\hline & 9 & 88.36 & 88.23 & -0.15 & 0.812 & 0.816 & 0.44 & 0.919 & 0.941 & 2.39 & 21.92 & 22.18 & 11.6 \\
\hline & 10 & 87.43 & 88.33 & 1.03 & 0.825 & 0.829 & 0.32 & 0.950 & 0.951 & 0.11 & 22.36 & 22.53 & 0.77 \\
\hline & $R M S$ & & & 1.66 & & & 6.14 & & & 3.18 & & & 4.96 \\
\hline
\end{tabular}

\section{DISCUSSION}

Table 1. presents the optimum performances of the implements under different soil types/ conditions. Results of this table revealed that the optimum speed of plough in clay-loam soil was $6 \mathrm{~km} / \mathrm{h}$. At this speed, the plough recorded the highest field efficiency of $88.11 \%$ at a cutting depth of $25 \mathrm{~cm}$ within soil moisture content range of $15.3-17.2 \%$ (w.b). In loamy - sandy soil, the optimum speed of the plough was also $6 \mathrm{~km} / \mathrm{hr}$ with corresponding field efficiency of $87.55 \%$ at moisture content range of $15.2-16.2 \%$ (w.b); while in sandy - clay soil, the plough recorded its optimum speed of $7 \mathrm{~km} / \mathrm{hr}$ with field efficiency of $87.78 \%$ at moisture content range of $14.8-18.6 \%$ (w.b).

Furthermore, the optimum speed of operation of harrow in clay-loam soil was $8 \mathrm{~km} / \mathrm{hr}$ with highest field efficiency of $87.98 \%$ within soil moisture content range of $14.2-16.2 \%$ (w.b). In loamy - sandy soil, the optimum speed of the harrow was $8 \mathrm{~km} / \mathrm{hr}$ with highest field efficiency of $87.19 \%$ at moisture content range of $13.3-15.4 \%$ (w.b); while in sandy - clay soil; it recorded its optimum speed of $9 \mathrm{~km} / \mathrm{hr}$ with the field efficiency of $98.54 \%$ at moisture content range of $13.0-19.3 \%$ (w.b). Results of this table also revealed that for all the soils studied, the optimum speed of ridger was $9 \mathrm{~km} / \mathrm{hr}$ with corresponding field efficiencies of $87.96 \%, 87.95 \%$ and $89.09 \%$ respectively in clay-loam, loamy-sandy and sandy clay soil at soil moisture content range of 14.0 $14.4 \%$ (w.b) , $13.2-14.5 \%$ (w.b) and 13.0 - 17.1\% (w.b) respectively. More so, the rotovator in recorded the same optimum speed with the ridger $(9 \mathrm{~km} / \mathrm{h})$ for all the soils with field efficiency of $89.81 \%$ at soil moisture content range of $13.0-14.2 \%$ (w.b) in clay-loam soil; $87.11 \%$ at moisture content range of $13.1-13.6 \%$ (w.b) loamy-sandy soil; and $89.40 \%$ at moisture content range of $13.3-16.3 \%$ (w.b) in sandy-clay soil.

Table 2 to 5 showed the regression equations developed from the experimental results obtained during the performance evaluation of the implements under study. 
The developed regression equations were validated by comparing its results with the experimental results using percentage error (Table 6 - 9). The comparison of the predicted results with the experimental results of this study revealed that the regression equations broadly did not over or under- predict the experimental results, thus, the prediction errors were within allowable range. More so, from the root mean square error analysis, the errors are within acceptable limit of $\pm 5 \%$. However, the little deviations in the prediction of some performance indicators in some operations were attributed to variations in soil conditions/characteristics. The coefficient of determination $\mathrm{R}^{2}$ for the regression equations developed for predicting the various performance indicators of the tractor - hitched implements vary from 0.7 to 0.9 which indicate that the equations are adequate for predicting the performances of the implements.

\section{CONCLUSION}

1. The optimum speed of plough in clay-loam and loamy - sandy soil was $6 \mathrm{~km} / \mathrm{h}$ with corresponding field efficiencies of $88.11 \%$ and $87.55 \%$ respectively, while in sandy - clay soil, the plough recorded its optimum speed of $7 \mathrm{~km} / \mathrm{h}$ with field efficiency of $87.78 \%$.

2. The optimum speed of harrow in clay-loam soil was $8 \mathrm{~km} / \mathrm{hr}$ with field efficiency of $87.98 \%$. In loamy - sandy soil, its optimum speed was $8 \mathrm{~km} / \mathrm{hr}$ with field efficiency of $87.19 \%$; while in sandy - clay soil; it recorded optimum speed of $9 \mathrm{~km} / \mathrm{hr}$ with field efficiency of $98.54 \%$.

3. The optimum speed of ridger was $9 \mathrm{~km} / \mathrm{hr}$ for all the soils with corresponding field efficiencies of $87.96 \%, 87.95 \%$, and $89.09 \%$ respectively, for clay-loam, loamysandy and sandy -clay soil.

4. The optimum speed of rotovator was also $9 \mathrm{~km} / \mathrm{hr}$ in all the soils with corresponding field efficiencies of $89.81 \%, 87.11 \%$, and $89.40 \%$ in clay-loam, loamy-sandy and sandy -clay soil, respectively.

5. The comparison of the predicted results with the experimental results (using percentage error and error root mean square) revealed that the regression equations broadly did not over or under- predict the experimental results, thus, the prediction errors were within allowable range of $\pm 5 \%$.

6. The coefficient of determination $\mathrm{R}^{2}$ for the regression equations developed for predicting the various performance indicators of the tractor - hitched implements vary from 0.7 to 0.9 which indicate that the equations are adequate for predicting the performances of the implements.

\section{RECOMMENDATIONS}

Differences exist in soil conditions among different agricultural or ecological areas; it is therefore recommended that more studies should be conducted in every agricultural zone to provide data on machine/ implement performances based on soil conditions for increased production, minimization of production costs, reduce loss/wastage of energy, time and waste of agricultural products. 
Finally, this study did not cover all the agricultural field machineries. Researchers are also recommended to make detailed time study in other machineries not covered in this work in other to provide database in their performances as to guide farmers here and other agricultural zones in machine/implement selections.

\section{REFERENCES}

[1] Gbadamosi,L. and Magaji, A. S. (2003). Field study on animal draft for farming in Zeguma village of Niger State. Proceeding of NIAE.

[2] Kual, R.N and Egbo, C. O. (1985). Introduction to agricultural mechanization, $1^{\text {st }}$ edition, published by Mac. pubisher. Ltd,. London and Basingstoke, pp.71-72.

[3] Oduma, O., Igwe, J E. and Ntunde, D. I. (2015). Performance evaluation of field efficiencies of some tractor drawn implement in Ebonyi State. IJET,UK. Vol. 5, No 4. Centre of professional research publications.

[4] Anazodo, U.G. N. (1983). Survey and comperative analysis of farm machinery management system in Nigeria. Proceeding of NSAE, VOL.7, pp. 59-73.

[5] Omar A. A., Ahmed E. M. E. and Sirelkhatim K. A. (2015) Performance of Disc and Chisel Ploughs and their Effects on Some Soil Physical Properties U. of K. J. Agric. Sci. 23(1), pp. 16-32, 2015.

[6] Sale, S. N.,Gwarzor, M. A., Felix, O. G and Idris, S. I. (2013). Performance evaluation of some selected tillage implements. Proceeding of NIAE. Vol.34.pp71-77.

[7] Saeed, R., Mohammad, A., Javad, J. (2015). Performance of Tractor and Tillage Implements in Clay Soil. Journal of the Saudi Society of Agricultural Sciences.Accepted article for publication.

[8] Olatunji. O.M. (2011). Evaluation of Plough Disc Performance on Sandy Loan Soil at Different Soil Moisture Levels. Research Journal of Applied Sciences, Engineering and Technology 3(3): 179-184.

[9] Oduma O., S. I. Oluka and P. C. Eze (2018). Effect of soil physical properties on performance of agricultural field machinery in south eastern Nigeria. CIGR Journal, 20(1): pp. $25-31$

[10] Afzalina, S., Shaker, M. and Zare, z. (2006). Performance evaluation of common drills in Iran. Canadian bio system engineering/lee genie des dio system es au canda. 48: 2:39-43

[11] Hunt, D. (2013). Farm power and machinery management. Tenth edition. Scientific International congress on agricultural engineering, agriculyural engineering, Dubli. Vol.3, pp.1703-1709.

[12] Kepner, R. A, Bainer, R and Barger, E. L. (1982). Principles of Farm Machinery, Avi publishing company Inc. Wester port.

[13] Onwualu, A.P., Oluka S.I. and J.C Adama (1998). Engineering opportunities for improvement of traditional practices for root crops in the former Enugu State. Journal of scince and technology. vol 4. pp. $23-35$.

[14] Kothari, C.R. (2014). Research Methodology. Methods and Techniques. Second Revised Edition. New Age International Publishers. New Delhi. pp. 140 - 145 


\title{
JEDNOSTAVNE REGRESIJSKE VEZE ZA PROCENU PERFORMANSI IZABRANIH PRIKLJUČAKA OPREME U JUGOISTOČNOJ NIGERIJI
}

\author{
Okechukwu Oduma $^{* 1}$, Chineze, G. Okeke ${ }^{2}$, Brendan E. Eje ${ }^{2}$, \\ Goziechukwu Inekwe ${ }^{1}$, Daniel C. Nnadi ${ }^{3}$ \\ ${ }^{1}$ Department of Agricultural \& Bioresources Engineering, \\ Michael Okpara University of Agriculture, Umudike, Nigeria \\ ${ }^{2}$ Department of Agricultural \& Bioresources Engineering \\ Enugu State University of Science and Technology \\ ${ }^{3}$ Department of Mechanical Engineering, \\ Michael Okpara University of Agriculture, Umudike; Nigeria
}

Sažetak: Istraživanje je obavljeno na tri dominantna poljoprivredna zemljišta jugoistočne Nigerije da bi se razvio određeni empirijski regresijski odnos za predviđanje performansi nekih odabranih priključaka za obradu zemljišta agregatiranih na traktore na osnovu eksperimentalnih rezultata dobijenih na terenu. Rezultati eksperimenata pokazuju su da optimalna brzina oranja u glinovito -ilovastom i ilovasto - peskovitom zemljištu iznosi $6 \mathrm{~km} / \mathrm{h}$, sa odgovarajućom efikasnosti (učinkom) na polju od 88,11\%, odnosno $87,55 \%$, dok je u peskovito- glinovito zemljištu za plug registrovana optimalna brzina $7 \mathrm{~km} / \mathrm{h}$ sa efikasnosti (učinkom) na polju od 87,78\%. Optimalna brzina operacije obrade drljanjem na glinovito-ilovastom zemljištu bila je od $8 \mathrm{~km} / \mathrm{h}$, a učinak (efikasnost) je 87,98\%. Kod ilovasto-peskovitog zemljišta, optimalna brzina bila je 8 $\mathrm{km} / \mathrm{h}$, a učinak (efikasnost) bila je $87,19 \%$; dok je u kod peskovito - glinovitog zemljišta registrovana optimalna brzinu od $9 \mathrm{~km} / \mathrm{h}$ sa učinakom (efikasnost) od 98,54\%.

Optimalna brzina traktor-podrivač bila je $9 \mathrm{~km} / \mathrm{h}$ za sva ispitivana zemljišta sa odgovarajućom efikasnosti (učinkom) na polju 87,96\%, 87,95\%, odnosno 89,09\%, za glinovito-ilovasta, ilovasto-peskovita i peskovito-ilovasta zemljišta. Optimalna brzina rotatofreze bila je takođe $9 \mathrm{~km} / \mathrm{h}$ na svim zemljištima $\mathrm{u}$ ispitivanju, sa odgovarajućom efikasnosti (učinkom) od 89,81\%, 87,11\%, odnosno 89,40\%, u glinovito-ilovastom, ilovasto-peskovitom i peskovito-glinovitom tipu zemljišta.

Ove efikasnosti (učinci) na polju su eksperimentalno dobijene na terenu su upoređene sa efikasnosti dobijenom iz jednačina regresija, koristeći grešku od $\pm 5 \%$. , a tačnost predviđanja testirana je korišćenjem srednjeg kvadratnog odstupanja $\mathrm{R}^{2}$. Upoređivanje predviđenih rezultata sa eksperimentalnim rezultatima otkrilo je da regresione jednačine $u$ velikoj meri nisu premašile ili umanjile eksperimentalne rezultate, tako da su greške predviđanja bile u okviru dozvoljenog raspona od $\pm 5 \%$.

*Corresponding Author. E-mail: odumaoke@gmail.com 
Koeficijent $\mathrm{R}_{2}$ za regresione jednačine razvijen za predviđanje različitih pokazatelja performansi traktorskih priključaka varira od 0,7 do 0,9 što pokazuje da su testirane varijable bile visoko povezane, a takođe su pokazatelj da su regresione jednačine adekvatne za predviđanje performanse priključaka.

Ključne reči: Empirijski, jednačine, performanse, regresija, primena obrade.

Prijavljen:

Submitted:

Ispravljen:

Revised:

Prihvaćen:

Accepted:
02.12.2019.

10.01.2020.

12.01.2020. 